\title{
INVESTIGATION OF ANGULAR DISTRIBUTIONS IN THE INTERACTION OF COSMIC-RAY PARTICLES WITH A DENSE TARGET AND COMPARISION WITH DATA OF THE LARGE HADRON COLLIDER
}

\author{
N.N.Zastrozhnova, O.A. Novolodskaya, N.S.Pokrovsky, T.Kh. Sadykov, \\ Y.M. Tautayev ${ }^{*}$, S.Zh. Tokmoldin \\ Institute of Physics and Technology \\ Almaty, Kazakhstan \\ E-mail: turlan43@mail.ru, tautaev@mail.ru
}

\begin{abstract}
The events registered in REC located in Tien-Shan mountain scientific station of cosmic rays at an altitude of $3340 \mathrm{~m}$ have been processed using the method of estimating probability density proposed at LHC. Total amount of registered events is 10199,462 events were selected of gamma ray families with $n \geq 4$. Gamma ray families with short avalanches in the ionization calorimeter in further analysis are not included. The paper presents received results of twoparticle angular correlations over a wide range of pseudorapidities $(\eta)$ and azimuth angle $(\varphi)$ and compared with data of the two-particle angular correlation on CMS detector in LHC.
\end{abstract}

The 34th International Cosmic Ray Conference,

30 July- 6 August, 2015

The Hague, The Netherlands

$$
\text { * Speaker. }
$$




\section{INTRODUCTION}

Measurements of angular two-particle correlations in proton-proton collisions (pp) to the center of mass energy contain important information characterizing quantum chromodynamics (QCD) in the power mode. In particular, the mechanism of hadronization and possible collective effects due to the high density of particles, that can be achieved in such collisions. Many particle correlations in high-energy collisions were measured earlier for a wide range of collision energies, and colliding particles in order to understand the mechanism underlying formation of particles. Development of method of studying cosmic rays interaction with matter having the fundamental importance was the creation of theory of nuclear-electromagnetic cascades in atmosphere and in dense layers of a substance that has allowed understanding the nature of TC and interpreting the data field research. An important feature of our experiment was the following: investigated interaction takes place in a dense target located at a height of 11 meters above the installation and all the REC exposure conducted jointly with a nuclear emulsion, which allowed us to investigate the fine structure of nuclear interactions (micron accuracy). The presence of a dense target enable to exclude many ambiguities typical for experiments studying atmospheric interactions, such as the unknown nature of target nucleus, height of interaction and therefore uncertainty in the measurement of angular characteristics, values of transverse momenta, etc.

Studies of interactions of cosmic ray particles with a dense target using X-ray emulsion chambers (REC) have been carried out on the high altitude research station of cosmic rays, located at an altitude of $3340 \mathrm{~m}$ in the mountains of Zailyisky Alatau in the pass Dzhusaly-Kezen [1,2]. REC method is based on X-ray emulsion detectors that enable to collect statistics for determined time period. The main object of study is a group of genetically related particles (gamma rays and hadrons) with sufficiently high energy, which result from interaction with solid target or with air nuclei of primary high-energy particles. These groups of particles called families.

To determine azimuthal anisotropy in interaction of cosmic-ray particles two-particle correlations were used, which is a powerful tool for studying the formation mechanism of particles in high-energy collisions of hadrons and nuclei. These studies provide important information characterizing quantum chromodynamics (QCD) in the power mode, especially hadronization mechanism and possible collective effects due to the particles high density, which can be achieved in such collisions.

\section{Selection of events generated in target.}

Mostly families of gamma rays generated in target were selected during process of analysis, and only in individual. Only a sample of target interactions greatly impoverishes the statistics, since the small target thickness as compared to the proportion of target with the atmosphere thickness, part of events in statistical material is small. However, selection of target interactions removes weight of uncertainties associated with analysis of atmospheric interactions.

The matter is that during studying of atmospheric gamma families registered by REC it is impossible to correctly determine the height of interaction point. Thus, all information relating to the transverse momenta of gamma rays, secondary impurities of nuclear interactions in atmosphere, the net contribution of electron-photon cascades obtained with a high degree of uncertainty. In addition, the analysis of atmospheric hadron-nuclear interactions remains uncertain the nature of target nucleus, is the collision occurred with the atom nucleus of $\mathrm{H}, \mathrm{N}, \mathrm{O}$ or Fe. 
The presence of a thin target and collecting of information from the ionization calorimeter considerably eliminates these problems and allows more accurate analysis of hadron-nuclear interactions.

Target interactions in the experiment were selected using the following criteria:

1. Number of gamma rays in the family $\mathrm{n}_{\gamma} \geq 4$.

2. Trajectory of gamma rays passes through the target.

3. Gamma-ray image of the family must be located within a circle of radius $\mathrm{R} \leq 30 \mathrm{~mm}$. This requirement stems from the relatively small height of target and the limitations of transverse momentum of secondary particles.

4. Azimuth and zenith angles of gamma rays must be the same within the measurement errors.

5. Total energy of the gamma rays, that is, the energy of the gamma family should be equal to $\Sigma \mathrm{E}_{\gamma} \geq 4 \mathrm{TeV}$.

6. Within a circle of $50 \mathrm{~cm}$ radius there are no gamma rays with angles of departure, to coincide with the corners of gamma rays family. This requirement excludes the contribution from the families formed in atmosphere to an altitude of $5 \mathrm{~km}$ above the set-up.

7. Families of gamma-rays are associated with short avalanches in ionization calorimeter, in a further analysis is not included. These ionization calorimeter data can reliably separate the pure electron-photon cascade from atmosphere of gamma ray families from nuclear interaction. In case of nuclear interaction of gamma rays are accompanied with hadrons which are giving long avalanche in calorimeter. Net EPC from atmosphere forms a short avalanche; almost all energy is released in first three rows of ionization chambers, layered with lead. Therefore, gamma-rays families associated with short avalanches are not included in further analysis.

For subsequent selection of families caused by nuclear interactions in target, the height of their generation was determined in two ways [3].

The first method uses the assumption of the constancy of transverse momenta of secondary particles. Height can be found from the expression:

$$
H_{1}=\frac{\sum_{i=1}^{n} E_{i} \cdot r_{i}}{\left\langle P_{\perp \gamma}\right\rangle \cdot n_{\gamma}}
$$

where $\left\langle\mathrm{P}_{\perp \gamma}>=0,2 \mathrm{GeV} / \mathrm{s} ; \mathrm{n}_{\gamma}\right.$ - number of gamma-rays in the family; $\mathrm{r}_{\mathrm{i}}$-distance of $\mathrm{i}$-th quantum to the center of family; $\mathrm{E}_{\mathrm{i}}$-energy of $\mathrm{i}$-th quantum. Coordinates of energy weighted center were calculated from the relations: 


$$
X_{i}=\frac{\sum E_{i} X_{i}}{\sum E_{i}} ; \quad Y_{i}=\frac{\sum E_{i} \cdot Y_{i}}{\sum E_{i}}
$$

The second way is to combine pairs of gamma rays in neutral pions. Indeed, all of the gamma rays in family are decay products of $\pi^{0}$-mesons born in the act of interaction. The height of family generation is given by:

$$
H_{2}=\frac{l}{m_{\pi^{0}}} \cdot \sqrt{E_{\gamma 1} \cdot E_{\gamma 2}}
$$

where 1-distance between a pair of gamma rays, $\mathrm{m}_{\pi}{ }^{0}$-neutral pion mass.

Naturally, due to the high energy threshold of gamma rays registration in REC part of gamma rays from $\pi^{0}$ mesons decay goes under the threshold and not registered. Therefore, not all gamma rays in family are possible to combine into neutral pions.

\section{The angular distribution of gamma rays families.}

From a consideration of the distribution presented it is evident that it is divided into three families, separated from each other. The left side corresponds to small EPC heights, the central part grouped around target events and the right side with the families of atmospheric interactions. Thus, using information from ionization calorimeter and application of above criteria enable to select 2657 gamma rays families from 10,199 recorded events. Further, 462 events were selected at gamma rays families with $n \geq 4$. Twodimensional correlation functions as $\Delta \eta-\Delta \varphi$ were studied for these events. Where $\Delta \eta-$ difference by pseudorapidity $(\eta=-\ln (\tan (\theta / 2))$ with the polar angle, measured as the deviation from beam axis, $\Delta \varphi$ is the difference between azimuth angles of two particles.

To find two-dimensional correlation function in plane of $\Delta \eta-\Delta \varphi$ Gaussian kernel PDE can be application in Monte-Carlo generation with $\mathrm{N}$ events generated in a kdimensional parameter space. These events are distributed by some unknown PDF, $\mathrm{f}(\mathrm{x})$. A Gaussian kernel method carry out an assessment the value of the PDF at $x$ point by the summary of Gaussains that centered at the Monte Carlo generated points, $\left\{\mathrm{y}_{\mathrm{i}}, \mathrm{i}=1, \mathrm{~N}\right\}$ [4]:

$$
\mathrm{f}(\mathrm{x})=\frac{1}{N|V|^{\frac{1}{2}}(2 \pi h)^{k / 2}} \sum_{i}^{N} \exp \left[-\frac{d_{i}^{T} V^{-1} d_{i}}{2 h^{2}}\right]
$$

Where $d_{i}=\left(x-y_{i}\right), V$ is a covariance matrix, and $\mathrm{h}$ is an additional scaling factor

The distribution similar to distribution obtained in the ATLAS experiment (Figure 2) have been obtained after data (Figure 1) processing. [5] 


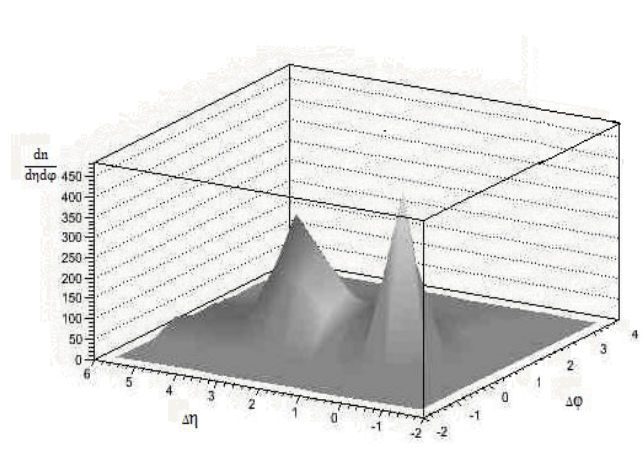

Figure 1 - the two-particle correlation function of pseudorapidity and azimuthal angles obtained in cosmic ray experiments

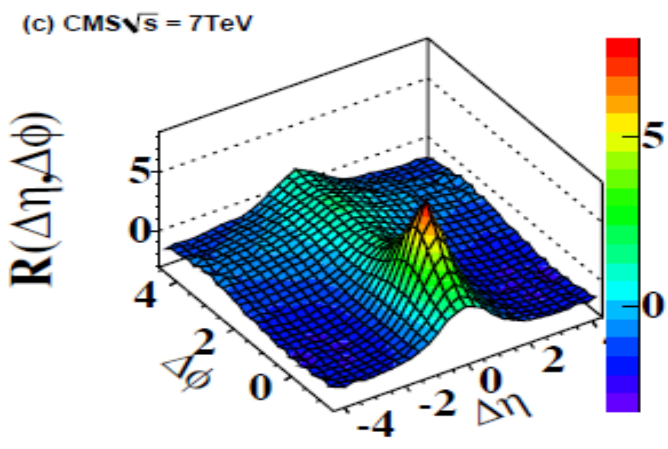

Figure 2 - the two-particle correlation function of pseudorapidity and azimuthal angles in PP-collisions in Atlas experiment

\section{SUMMARY}

As can it can be clearly seen from the figures 1 and 2 distinct structure in two-dimensional correlation function is observed in events with the high multiplicity. In comparison with result of two-dimensional correlation function in PP interaction at energy of $7 \mathrm{TeV}$ in the LHC (Figure 1) should be noted that the resulting data have a good similarity with the twodimensional data correlation function in cosmic rays (Figure 1). It can be concluded that in cosmic rays at approximately the same energies mainly protons are detected interacting with such light material as graphite. In future, these structures will be described with a simple model of "independent clusters" in order to quantify their strength (cluster size) and distribution of $\eta$ (width of cluster decay). This is the first observation of such a structure in two-particle correlation function of cosmic ray particles interaction with matter.

\section{References}

[1] N.A Dobtoyin, A brief history of the first period of field work of cosmic rays at the Physics Institute of the USSR, FIAN, Moscow 1998

[2]. G.T. Zacepin, Nuclear cascade process and its role in the development of broad atmosfer showers // DAN USSR, 1949, T. 67, P. 993

[3] Sadykov T.Kh., Determining the height of the interaction of hadrons to install Hadron44" // AGU Abay. 2003, №1 (7), P. 200-201.

[4] S. Towers, Kernel probability density estimation methods, State University of New York at Stony Brook.

[5] Observation of Long-Range, Near-Side Angular Correlations in Proton-Proton Collisions at the LHC. The CMS Collaboration_arXiv:1009.4122v1 [hep-ex] 21 Sep 2010. 